\title{
Hands-on Exposure to Unconventional Applications of Aerospace at the High School Level
}

\section{Ms. Hannah Stroud}

Hannah Stroud is a first year masters student at Texas A\&M University. Her research interests include unconventional applications of aerospace technology with an emphasis on smart materials and structural optimization. She is co-advised by Dr. Kristi Shryock and Dr. Darren Hartl.

\section{Dr. Kristi J. Shryock, Texas A\&M University}

Dr. Kristi J. Shryock is the Frank and Jean Raymond Foundation Inc. Endowed Instructional Associate Professor and Associate Department Head in the Department of Aerospace Engineering in the College of Engineering at Texas A\&M University. She also serves as Director of the Craig and Galen Brown Engineering Honors Program. She received her BS, MS, and PhD from the College of Engineering at Texas A\&M. Kristi works to improve the undergraduate engineering experience through evaluating preparation in areas, such as mathematics and physics, evaluating engineering identity and its impact on retention, incorporating non-traditional teaching methods into the classroom, and engaging her students with interactive methods. 


\title{
Hands-on Exposure to Unconventional Applications of Aerospace at the High School Level
}

\begin{abstract}
For Institution, STEM education at a high school level and enrollment of a diverse population of students have long been priorities. The aerospace engineering department at Institution has implemented programs to address those priorities within the discipline itself. Camp SOAR, a week-long summer STEM camp for high school students interested in aerospace engineering, seeks to introduce aerospace concepts in an accessible way while providing hands-on building and learning experience to students. High school students are instructed in key engineering concepts and aerospace engineering fundamentals, which they then apply to a major project that they test in a competition at the end of the week. In addition, a new diversity initiative through the aerospace department at Institution seeks to investigate how perception of the aerospace discipline affects diversity within the department. The authors hypothesize that introducing non-traditional applications of aerospace knowledge will encourage students to apply aerospace concepts to a broader range of engineering problems and organically increase the number of students from underrepresented categories who are interested in the discipline. This hypothesis is herein tested on a small scale through an educational activity introduced in Camp SOAR. Students' perceptions of aerospace are evaluated before completing an "unconventional" aerospace activity. The students then complete the activity, and perception shift is evaluated.
\end{abstract}

\section{Introduction}

Since 2012, Texas A\&M University has placed a concentrated focus on outreach to high schools and sought to foster interest in aerospace engineering through Camp SOAR, a summer camp for high school juniors and seniors. Camp SOAR has prioritized effective communication of aerospace fundamentals through activity-based learning in a way that has been both captivating and instructive without being overwhelming to its audience [? ? ]. Students are separated into "tracks", project groups led by field specialists, through which the students have been able to explore their personal interests within aerospace; additionally, more individual project guidance can be given in this format. To accommodate growth from previous years, a fourth track, helicopters, has been added to the pre-existing airplanes, rockets, and air ships. These tracks promote exploration of aerospace in a very traditional capacity-that is, the projects focus on 
projects typically associated with aerospace. For example, students in the airplane track build a glider attached to a pneumatic-powered rocket, which is constructed by students in the rocket track. Students in the air ships track design a control scheme for lighter than air swimmers, and students in the helicopters group design and manufacture boomerangs.

A recent hypothesis from the authors states that a limited perception of aerospace engineering might negatively impact interest in the field. If students perceive aerospace as having more applications than traditional aerospace areas (planes, rockets, helicopters, satellites, airships), interest in aerospace engineering might increase. To test this hypothesis in a small-scale environment at an early education level, an activity related to a "non-traditional" aerospace region was introduced. In this group of students, interest in aerospace engineering was generally high; however, in a pre-activity evaluation, students tended to see aerospace as "limited" regardless of interest level. They also considered traditional aerospace applications, such as space exploration, to be more relevant to the field than less traditional applications, such as renewable energy or healthcare-related technology. To bridge that gap, students were given a prompt to design a capsule to protect a biomedical payload as it was airdropped over a deployment site, requiring them to consider the capsule's structure and aerodynamics. The students were given a budget, a list of available materials, and a cost function to drive their design. In an initial design session, students were asked to form a preliminary design and place an order for materials necessary to build their designs while remaining within their budget. In a second design session, students were tasked with using the materials they ordered to build their designs. The students loaded their designs with a "syringe" payload (glow sticks) and tested them at a drop height of six stories. From this activity, students learned the importance of system-level evaluation and designing to requirements while applying their aero intuition to an unconventional aerospace problem. Feedback from students showed promise of changing the perception that careers for aerospace engineers are limited to traditional areas.

\section{Camp Background}

For seven years, the aerospace engineering department at Texas A\&M University has held a week-long summer camp for students entering their junior and senior year of high school. With an intent for students to explore the major and learn more about aerospace engineering, the camp has trained almost 400 participants. The use of a selection protocol as well as an impartial review process has provided a straightforward means of attaining the objective of impartial selection for camp participants. Even with blind review of applications, percentages of women, students from groups underrepresented in engineering, and students from out-of-state have been higher than typically seen in the aerospace department at Texas A\&M. For example, the department has less than $20 \%$ women as undergraduates; however, the number of women participating in the 2018 camp was 33\%. This has not been an uncommon statistic through the years. Participants are grouped equally into one of four project design teams: rockets, aircraft, airships, or helicopters. While students provide their preferences during the application process, teams are ultimately determined by camp personnel. During the 2018 camp, women ended up being equally distributed among three of the four tracks: rockets, aircraft, and airships. A very low percentage of women were in the helicopters track. 
Rocket and Aircraft: The rocket and aircraft teams design and build a vehicle to launch a glider on its back and then release the glider to fly back to the ground. The project is structured to require the two tracks to form new teams consisting of rocket track members to build the rocket and aircraft track members to design and build the glider. The teams then compete on the basis of maximum time aloft. The task requires that the rocket students build a rocket to launch the system as high as possible, considering the rocket motor constraints. The aircraft team is required to design a glider that, once free of the rocket, remains in the air as long as possible. The longest flight time, from launch to glider landing, is the winner. While the rocket and the glider are designed and built separately, the design and placement of the mechanism that holds the glider on the back of the rocket is a team effort.

Airships: Students in the airship group utilize the popular air swimmers toy, a lightweight nylon bladder about four feet long, shaped and colored like a fish and filled with approximately 4.5 cubic feet of helium to make it buoyant. Each of these floating vehicles is outfitted with flappable tail for propulsion and a pitch control device consisting of a weight that a second small DC motor moves along a track to control pitch. The tasks for the students are twofold: 1) experiment with different tail shapes to determine best performance and 2) modify the air swimmer by adding an Arduino microcontroller connected to the tail motor to allow for autonomous flight. The Arduino is programmed using an in-house developed computer code that allows students to adjust the amplitude and frequency of the tail flapping. The objective is to determine the design parameters that permit the fastest completion of a given course.

Helicopters: Students in the helicopter track design and construct boomerangs out of 1/4" plywood. The students use CAD software (SolidWorks) to design the planform of their boomerang, receiving feedback on how certain aspects of their design will affect its flight performance. The designs are then laser cut, and students shape their boomerangs, sanding the leading and trailing edge appropriately. Students then test their designs by throwing them. The goal for this track is to manufacture the boomerang that returns closest to the point from where it was thrown.

\section{Incorporating Unconventional Applications of Aerospace}

\section{Unconventional Applications Project Background}

As the population of the US grows increasingly diverse[1], the lagging diversity numbers of STEM fields has become a concern at a national level [2, 3]. Fields perceived as "niche" and specialized often lack diversity. Historically, aerospace engineering in particular has been a discipline perceived as having only military and flight-vehicle related applications; however, this isn't necessarily the case. Aerospace engineering skill sets have been employed in renewable energy, data security, healthcare, and education, as the true aerospace engineering strengths reside in fluid mechanics, structural design, and dynamics and control. Prior research has shown how incorporating more communal applications of STEM fields can improve gender-representation [4], and many alternate applications of the aerospace field may appeal to a more diverse set of students. The authors hypothesize that if students are exposed to engineering problems uniquely 
solvable by an aerospace engineering skill set but that are not traditionally aerospace problems, they will be more likely to perceive more areas of engineering problems as related to aerospace.

\section{Activity Formulation}

As a method of testing the hypothesis, an activity which highlighted a non-traditional aerospace problem was developed and incorporated into the camp. The goal of this activity is twofold: first, the proposed activity would introduce the engineering concepts of design constraints, budgeting, and communicating with customers and suppliers; second, it would require the use of aerospace engineering concepts without being a project students typically would think of as "aerospace".

The activity designed to meet these goals is a payload drop problem. The activity prompt states that a small UAV is dropping medical supplies to a war-torn country- students must design a transfer pod to protect at least four syringes, simulated by glow sticks, that falls as quickly as possible. This incorporates two key aspects of aerospace engineering: structures, as the pod must not allow the payload to break, and aerodynamics, as the pod must fall quickly.

To introduce the engineering concepts, the activity is presented in phases. The first phase is a preliminary design phase. Students are given the prompt and instructed to form teams and develop a design concept that will win a contract bid with a customer. The customer provides a a budget as well as a cost function to drive design, placing various percent weights on the design aspects pod weight, material cost, and number of syringes carried, while also penalizing for any damaged payload. The cost function given is shown in equation 1 , and students were instructed to minimize the value of this function.

$0.3\left(\frac{\text { pod weight }}{\text { payload capacity }}\right)+0.5\left(\frac{\text { material cost }}{\text { budget }}\right)+0.2\left(\frac{4-\text { number of syringes }}{4}\right)+100_{\text {Ifdamagedpayload }}$

Four types of materials- styrofoam, balsa wood, paper, and 3D printed plastic- and their respective costs per unit volume are provided. The students must then use their design to develop a materials list and place an order with the supplier. The next phase is a design and build session. Materials ordered are delivered to the teams, and students begin executing their designs. Once built, designs are loaded with syringes and weighed. The final phase is the test phase, where students evaluate the structural integrity of their designs with a drop test from a height of six stories.

\section{Results}

Before the activity was introduced, students were asked to evaluate their perceptions of aerospace engineering and gage their interest level. Students were asked some basic demographics questions (reported in Camp Background), if they plan to major in engineering, the problem they were most 


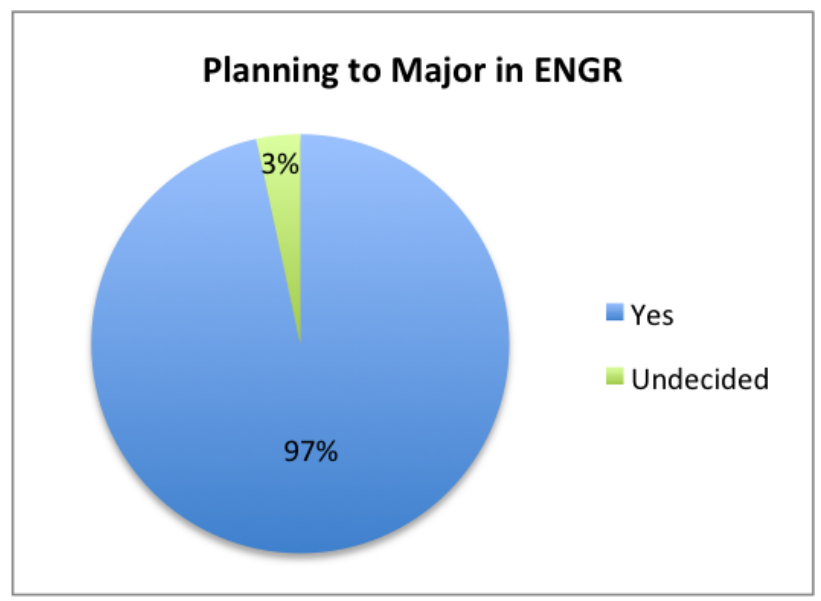

(a) Students interested in majoring in engineering

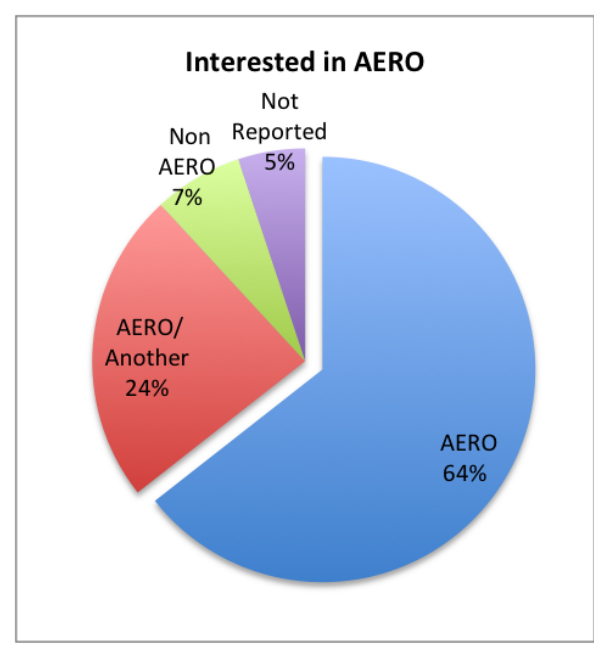

(b) Students interested in aerospace specifically

Figure 1: Results of survey for students interested in engineering and aerospace engineering.

interested in solving, and then to rank on a scale of 1-5, where 1 is not interested and 5 is most interested, their interest level solving problems in the following categories: energy, infrastructure, environment, healthcare, education, securing information, and space. Students were then asked to rank on that same scale whether or not they believed each of those topics to be related to aerospace engineering. They were then asked what they planned to major in upon attending college, what their motivation was for choosing this, and what their desired career was.

As demonstrated in figure 1a, a vast majority of students planned to major in engineering. Figure $1 \mathrm{~b}$ shows that $88 \%$ of students were considering aerospace as their major with $64 \%$ indicating it was their only choice; these numbers are consistent with a camp intended to attract students with an affinity for aerospace engineering.

Students reported their interest in topics with a non-obvious correlation to the aerospace industry. The results are normalized and sorted by gender (figure 2), with a two period moving average line plotted to indicate data trends. With such a high interest in aerospace in this group, the category most obviously related to the discipline, space, drew the highest interest. The most notable disparities in interest between gender occur in the infrastructure and healthcare categories. A higher percentage of female students indicated a non-interest in infrastructure, compared with the male students whose distribution was more normal. Conversely, male interest in healthcare trended toward the non-interest side, while female students trended toward the interest side.

Students then reported how related to aerospace engineering they believed these same topics to be (figure 3). Again, regardless of gender, students tended to classify space as very related. The categories of energy and the environment were both represented by trends towards more related, while healthcare and education were seen as more unrelated.

Interestingly, student interest in a topic often led to an increased belief that it was aerospace related; however, the inverse was not true, and students perceiving a topic as related to aerospace did not increase the level of interest. The exception to this was the space category, in which a vast majority of students were both very interested and saw the topic as related. 

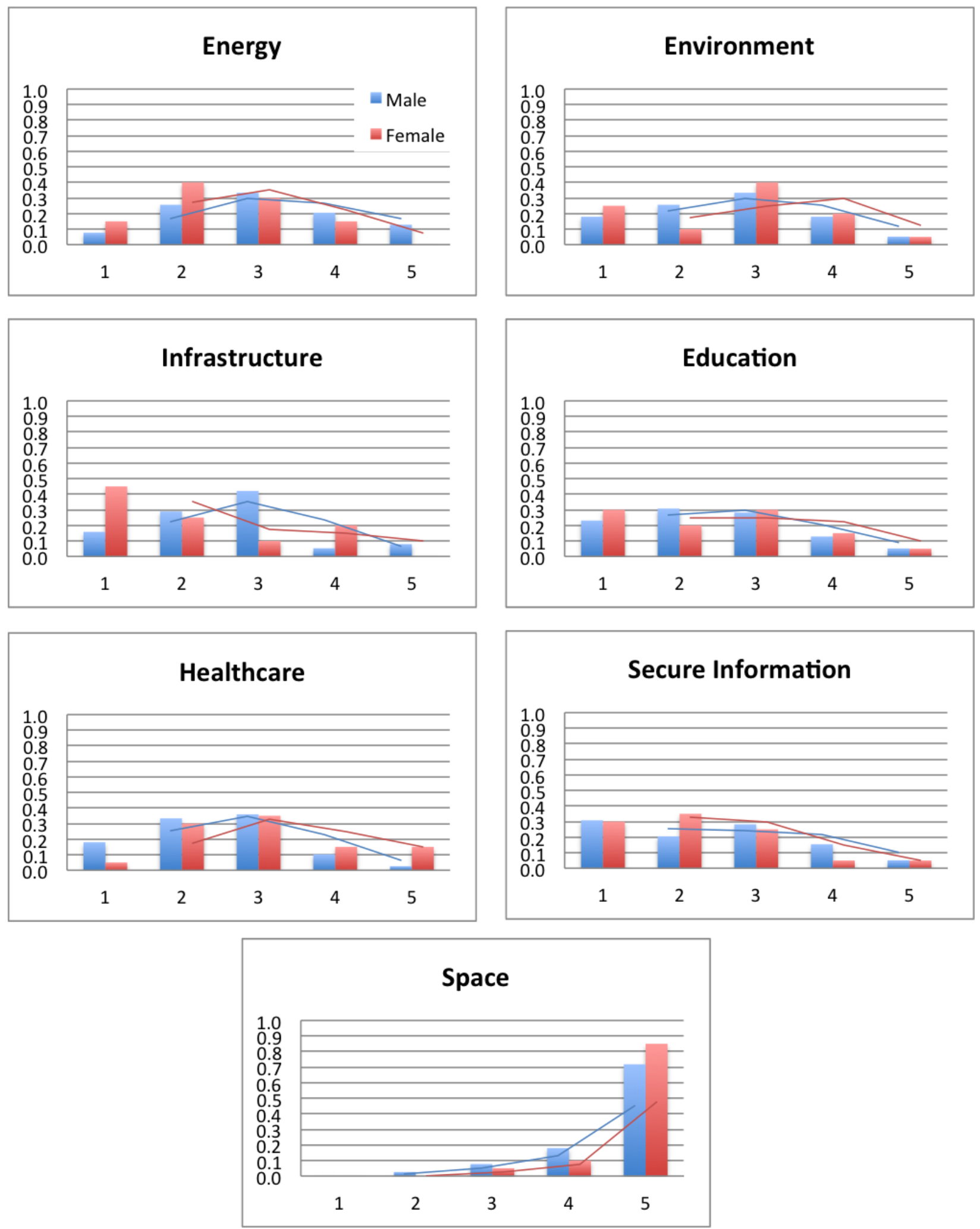

Figure 2: Normalized report of student interest in concepts sorted by gender. Blue indicates male, red indicates female. 

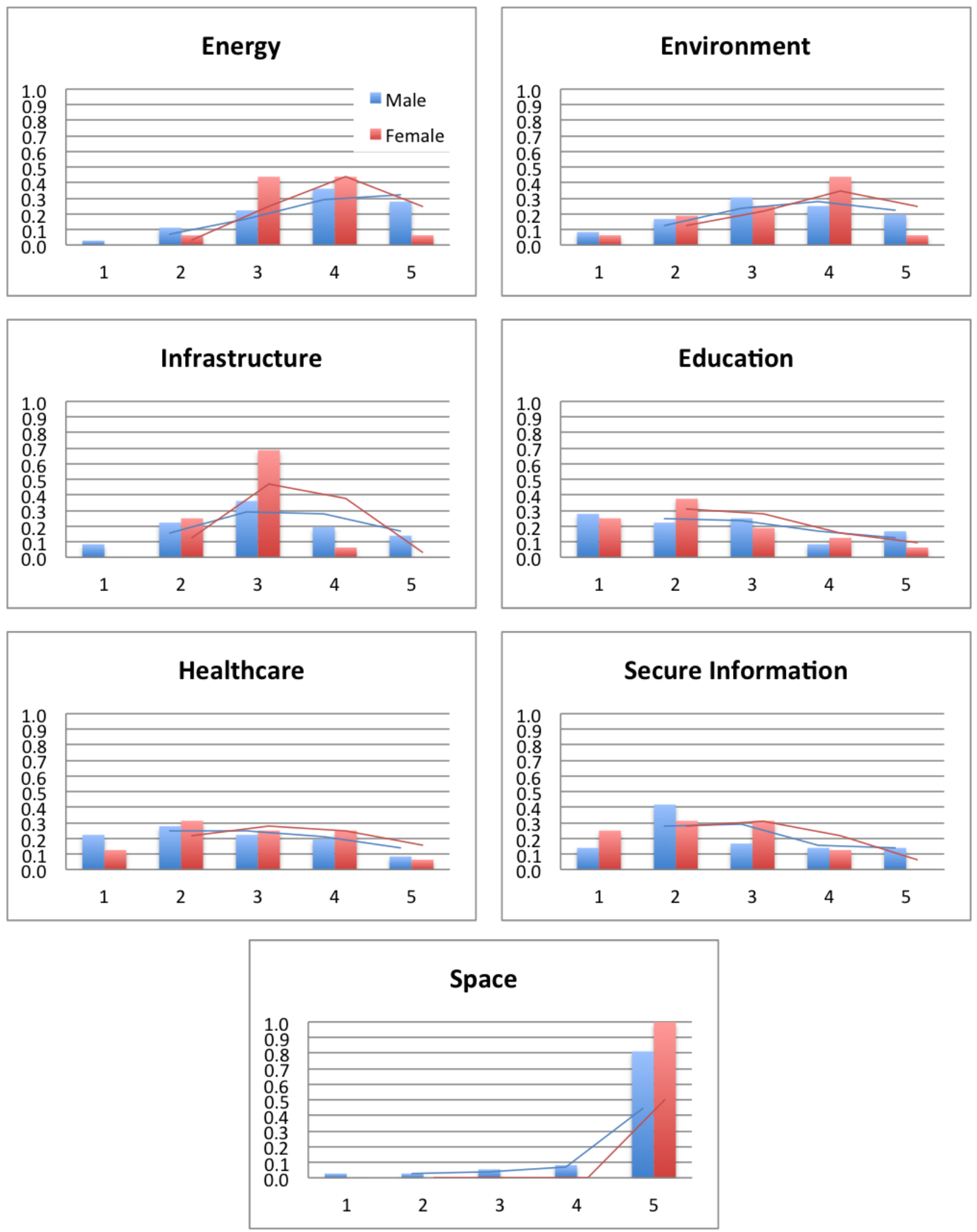

Figure 3: Normalized report of student perception of relation to aerospace of concepts sorted by gender. Blue indicates male, red indicates female. 


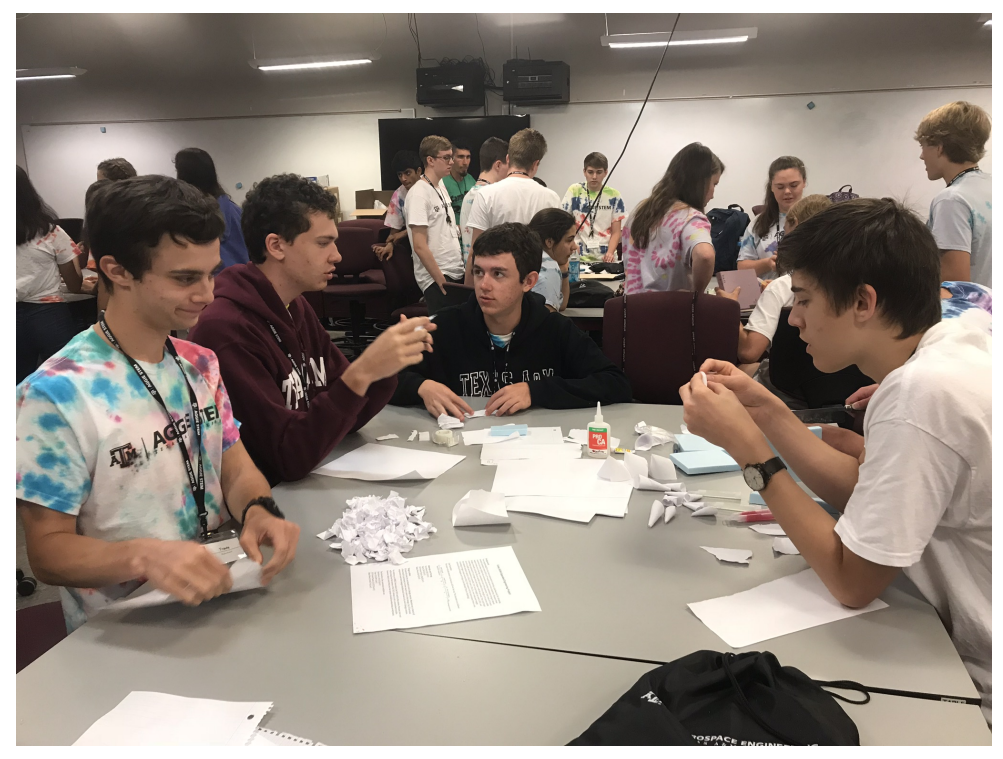

Figure 4: Students building their design.

After the initial data was taken, the first phase of the activity was introduced to the students. Students received the prompts, sorted themselves into teams, and proceeded to design their capsules and order their materials. All materials except sheets of paper were ordered by dimension to better allow students to estimate weights with simple volumetric calculations and provided material densities. Students were encouraged to keep the manufacturing process in mind during the design phase to preclude any attempts at overly ambitious designs for the allotted time. This process went smoothly, and all teams finished the design session with a sketch and a completed materials order. Pitfalls from this phase included students tending to concentrate on maximizing the number of syringes they were designing for despite material cost being the most heavily weighted factor in the cost function, as well as one team neglecting units, and therefore receiving a smaller amount of material than expected.

During the second phase, students received their materials and began building (figure 4). Immediately, students began to realize the difference between the amount of material they thought they needed and the amount actually required for their design. The only material they were allowed to order more of throughout the build phase was paper, which proved sufficient for most teams. Many teams iterated on their initial design in order to build a functional product with the materials they had in the time allotted. Once the designs were constructed, they were loaded with the payload (i.e. number of glow sticks requested by the team) and weighed.

Students then brought their designs to the testing area, where they proceeded to drop test their pods. A preliminary test was run where an unprotected glow stick was dropped from the test height to determine that it would break upon contact with the ground. After this test did in fact break the payload, students dropped their designs hoping for the opposite result. To determine the success of a drop, the pods were taken to a dark room and inspected for any glowing payload. Every team had a successful drop, meaning no glow sticks were cracked. Therefore, the competition winner came down to which team did the best in the cost function. The winning team used less than half of the amount of material than any other team, giving them a very low score. 


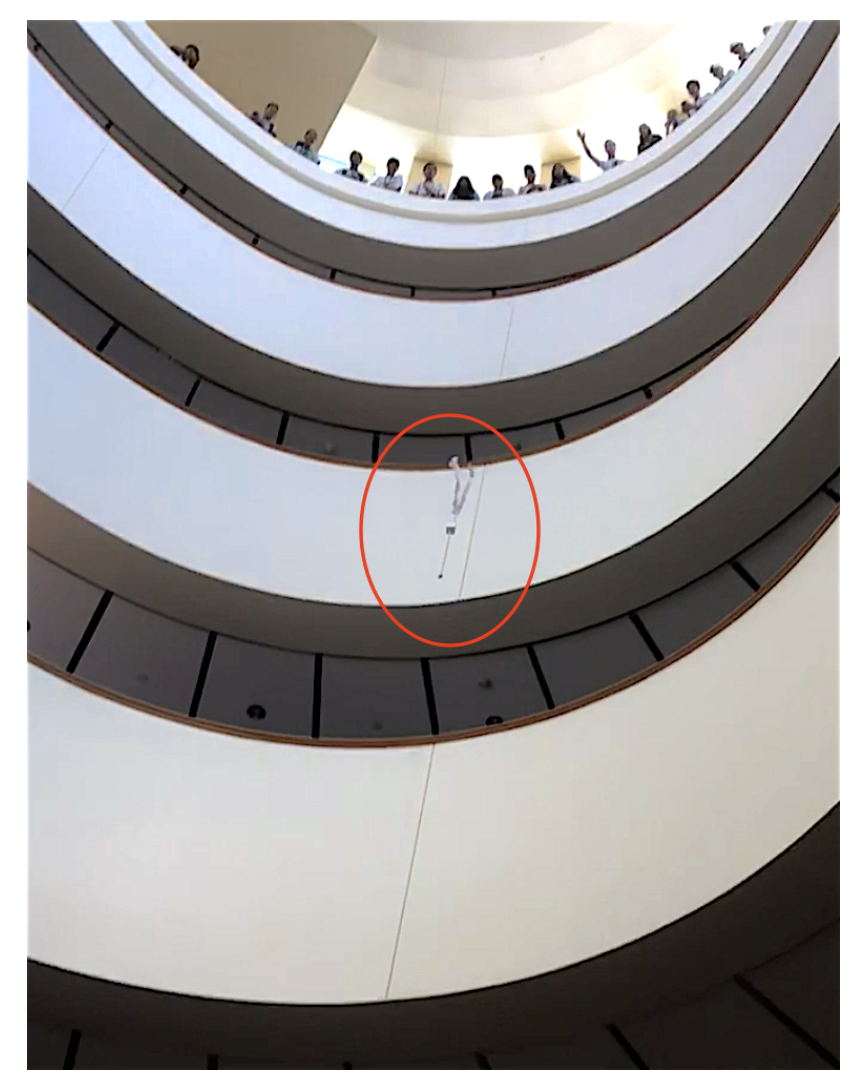

Figure 5: The winning team tests their design.

Their design used a sort of "pogo stick" to increase the time of the initial impact as well as strips of paper as "aerodynamic stabilizers" that ensured the pod landed pogo stick side down. This design can be seen during test in figure 5

Through this activity, students were exposed to key aspects of the engineering design process. Students learned the importance of budgeting, communication, and flexible design. Problems that arose from every phase of the activity resulted in additional engineering lessons: always include correct units, quantity estimation is challenging especially on a budget, and a good engineer must optimize a design for what the customer prioritizes most. Additionally, students that incorporated their aerospace intuition into their design were the most successful in the competition, thereby meeting the goal of this activity.

After the activity, students were asked to qualitatively assess whether the activity was related to aerospace, and if their perception of "aerospace related" activities was broadened by performing the activity. All students agreed that the activity both required aerospace knowledge and expanded what they would see as aerospace related problems. In addition, the activity was performed in a fun and engaging way that the students enjoyed. In a free response question asking which activities students enjoyed the most in a post-camp general survey, $16 \%$ of students mentioned this activity as their favorite, beating out a visit to a trampoline park, mentioned in only $13 \%$ of responses, and game nights, which were mentioned in $9 \%$. 


\section{Conclusions}

\section{Lessons Learned}

Though the activity was a success, many aspects can be improved upon for future iterations. For example, the short time allotted, approximately 45 minutes, for the activity led to a more rushed design session. Many of the challenges students experienced with the design and materials ordering process could have been mitigated if the students had more time to plan and complete them.

\section{Future Work}

Because the activity was both fun and educational for the campers, future iterations of Camp SOAR will include unconventional aerospace activities as well. Though future activities may not take the form of the payload drop, key aspects will be maintained, such as the hands-on build and test, the aerospace skills components, and the engineering concept introduction.

\section{Acknowledgments}

The authors would like to thank everyone involved in putting on Camp SOAR. Funding for this work is graciously provided by NSF Project 1730693.

\section{References}

[1] U.S.Census Bureau. Most children younger than age 1 are minorities, census bureau. URL https://www.census.gov/newsroom/releases/archives/population/cb12-90.html.

[2] National Academy of Sciences. Rising above the gathering storm: Energizing and employing america for a brighter economic future. The National Academy Press, Washington, DC.

[3] S. Olson and D.G. Riordan. Engage to excel: Producing one million additional college graduates with degrees. in science, technology, engineering, and mathematics. Report to the president. Executive Office of the President.

[4] C. Hill, C. Corbett, and A. St Rose. Why so few? Women in science, technology, engineering, and mathematics. American Association of University Women, Washington, DC. 